\title{
New Tetrahedral Global Minimum for the 98-atom Lennard-Jones Cluster
}

\author{
Robert H. Leary \\ San Diego Supercomputer Center, University of California, San Diego, San Diego, CA 92138, USA \\ Jonathan P. K. Doye \\ University Chemical Laboratory, Lensfield Road, Cambridge CB2 IEW, United Kingdom
}

(March 22, 2021)

\begin{abstract}
A new atomic cluster structure corresponding to the global minimum of the 98-atom LennardJones cluster has been found using a variant of the basin-hopping global optimization algorithm. The new structure has an unusual tetrahedral symmetry with an energy of $-543.665361 \epsilon$, which is $0.022404 \epsilon$ lower than the previous putative global minimum. The new LJ ${ }_{98}$ structure is of particular interest because its tetrahedral symmetry establishes it as one of only three types of exceptions to the general pattern of icosahedral structural motifs for optimal LJ microclusters. Similar to the other exceptions the global minimum is difficult to find because it is at the bottom of a narrow funnel which only becomes thermodynamically most stable at low temperature.
\end{abstract}

02.60.Pn,36.40.Mr,61.46.+w

The determination of the global minima of LennardJones (LJ) clusters by numerical global optimization techniques has been been intensely studied in the size range $N=13-147$ by both chemical physicists and applied mathematicians [1,2]. The LJ potential, which is given by

$$
E=4 \epsilon \sum_{i<j}\left[\left(\frac{\sigma}{r_{i j}}\right)^{12}-\left(\frac{\sigma}{r_{i j}}\right)^{6}\right]
$$

where $\epsilon$ is the pair well depth and $2^{1 / 6} \sigma$ is the equilibrium pair separation, is a simple yet reasonably accurate model of the interactions between heavy rare gas atoms. In general, there has been good agreement between physical measurements on rare gas clusters from electron diffractometry [3] and mass spectrometry [4] and computational global optimization results regarding magic number sizes and corresponding cluster geometries [6]. Both approaches find that Mackay icosahedra [7] are the dominant structural motif.

The LJ microcluster problem has also become a benchmark for evaluating global optimization algorithms. The number of local minima (excluding permutational isomers) on the potential energy surface (PES) is believed to grow exponentially with $N$ [8,9] and is estimated to be of the order of $10^{40}$ for $N=98$. A wide variety of global optimization techniques including simulated annealing [10], genetic algorithms 11 13., smoothing and hypersurface deformation techniques [14,15, lattice methods [6, 16], growth sequence analysis [17,18], and tunneling [19] have been applied to the problem. Unbiased methods that make no assumptions regarding cluster geometry are of the most interest, since these have the best chance of successful generalization to more complex potentials such as those in the protein folding problem.

Most of the global minima in this size range were first found by Northby in a lattice-based search of icosahedral structures [6]. These structures consist of a core Mackay icosahedron (Figure 1 1b) surrounded by a partially filled outer shell. More recently, there have been a number of improvements in some of these putative global minima. Firstly, further refinements to Northby's algorithm, particularly the relaxation of the assumption that the core Mackay icosahedron is always complete, has a led to a number of new global minima [16, 18,20,21. Secondly, consideration of particularly stable face-centredcubic (fcc) and decahedral forms has also led to new global minima [19,22,23. At $N=38$ the global minimum is a fcc truncated octahedron (Figure 1 $1 \mathrm{a}$ ) and at $N=75$ 77 and 102-104 the global minima are based on Marks decahedra (Figure 11c). Thirdly, powerful unbiased global optimization algorithms, particularly the basin-hopping [24] and genetic algorithms [12,13], have recently begun to catch up with those methods that incorporate particular physical insights into the LJ problem, and are now able to find all the known lowest-energy minima.
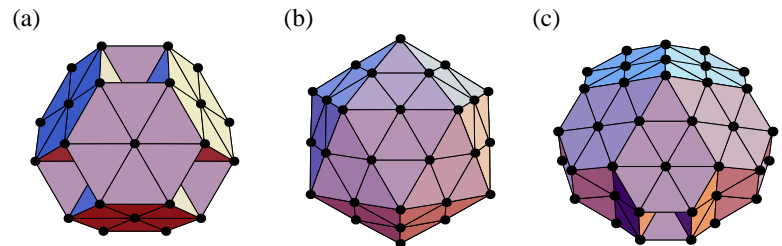

FIG. 1. Three particularly stable examples of the known morphologies for LJ clusters: (a) the 38-atom fcc truncated octahedron, (b) the 55-atom Mackay icosahedron, and (c) the 75-atom Marks decahedron.

Given this combined attack on the LJ optimization problem, it might have been imagined that all the global minima for $N<150$ had been found. Here, however, we report a new lowest-energy structure for $\mathrm{LJ}_{98}$, which has an energy of $-543.665361 \epsilon$ and $T_{d}$ point group symmetry. This compares to an energy of $-543.642957 \epsilon$ for the previous icosahedral putative global minimum which 
was found by Deaven et al 12 . The $\mathrm{LJ}_{98}$ global minimum is organized around a central fcc tetrahedron with four atoms on each edge (Figure 2c). Four additional fcc tetrahedrons (minus apices) are erected over the faces of the central tetrahedron to form a 56-atom stellated tetrahedron (Figure 2 $\mathrm{b}$ ). An additional 42 atoms decorate the closed-packed sites on the surface of the stellated tetrahedron to complete the structure (Figure 2 a). The new $\mathrm{LJ}_{98}$ structure is of particular interest because its tetrahedral symmetry establishes it as only the third known type of exception to the general pattern of icosahedral structural motifs for optimal LJ microclusters, and the first to be discovered by an unbiased optimization method.

Given its unusual structure one might wonder why it is so low in energy. For LJ clusters optimizing the energy is a balance between maximizing the number of nearest neighbours and minimizing the strain energy (the energetic penalty for nearest-neighbour distances deviating from the equilibrium pair value) [22]. The spherical shape and high proportion of $\{111\}$ faces gives the structure a large number of nearest neighbours (432 compared to 437 for the lowest-energy icosahedral minimum and 428 for the lowest-energy decahedral structure), whilst its strain energy is intermediate between icosahedral and decahedral structures. The lower strain energy allows it to be lower in energy than the icosahedral minima, even though it has fewer nearest neighbours. The strain in the structure is focussed around the six edges of the central fcc tetrahedron. The atoms along these edges have the same local coordination as atoms along the five-fold axis of a decahedron.

(a)
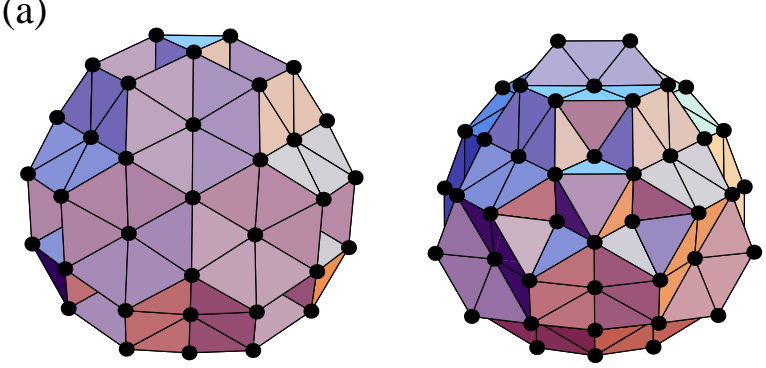

(b)

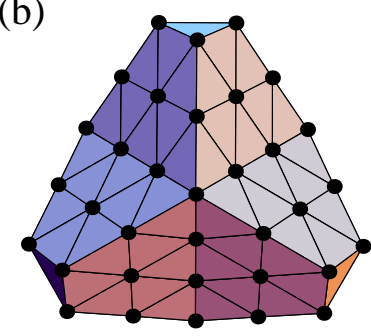

(c)

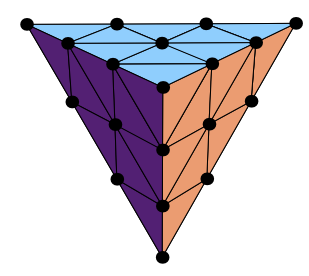

FIG. 2. (a) Front and back views of the new $\mathrm{LJ}_{98}$ global minimum. (b) The 56-atom stellated tetrahedron and (c) the 20 -atom tetrahedron that are at the centre of this structure.
It is also natural to ask how general this structure is. Firstly, analogous structures can be formed with smaller and larger tetrahedra at their core. The previous one in this series is at $N=34$ and the next one is at $N=195$. However, these structures are not energetically competitive: the former because it has too high a proportion of $\{100\}$ faces, and the latter because it is not sufficiently spherical. Secondly, the structures of the other non-icosahedral LJ global minima have been experimentally observed for gold [25] and nickel [26] clusters, and found to be particularly stable in theoretical calculations of transition metal clusters [27]. Therefore, we performed some optimization calculations for the Sutton-Chen family of potentials [28]. The tetrahedral structure was lowest in energy for silver, but a decahedral minimum was lower in energy for nickel and a fcc minimum for gold. This is consistent with previous results, which indicted that, of these three metals, silver clusters exhibited ordered structures with the most strain [27.

The new $\mathrm{LJ}_{98}$ optimum was found using a variant of the basin-hopping global optimization algorithm [24]. The key idea behind the algorithm is the mapping of the original LJ potential energy function, $E(\mathbf{x})$, for each point $\mathbf{x}$ on the $3 N$-dimensional Cartesian coordinate space onto a "transformed" energy function, $T(\mathbf{x}) . T(\mathbf{x})$ takes the value of $E(\mathbf{x})$ at the local minium, $\mathbf{x}_{\mathbf{m i n}}$, arrived at by applying a given local optimization procedure, such as the conjugate gradient algorithm, with $\mathbf{x}$ as the starting point for the algorithm. Thus $T(\mathbf{x})$ is a "plateau" function that takes on the constant value $E\left(\mathbf{x}_{\mathbf{m i n}}\right)$ on the catchment basin surrounding each local minimum $\mathbf{x}_{\min } . T(\mathbf{x})$ is a lower bound to $E(\mathbf{x})$ and coincides with $E(\mathbf{x})$ at all of the latter's local minima, but all barriers are removed in the $T(\mathbf{x})$ landscape and transitions bewteen catchment basins can take place all along the basin boundaries.

The original basin-hopping algorithm consists of a Metropolis search of the transformed landscape, $T(\mathbf{x})$, using a Monte Carlo sampling procedure to move between local minima. In the variant used in the discovery of $\mathrm{LJ}_{98}$ 29], the Metropolis criterion of accepting uphill moves with a probability that is an exponentially decreasing function of the energy increment is abandoned in favor of only accepting downhill moves. The algorithm is restarted from a fresh random starting local minimum whenever progress stalls for a sufficiently large number of move attempts. The variant was successful in locating the $\mathrm{LJ}_{98}$ global minimum in 6 of 1000 random starts, with a mean computational time between encounters of about 30 hours on a $333 \mathrm{MHz}$ Sun Ultra II processor. This structure has also been subsequently found using the original basin-hopping algorithm [30].

These results show that the $\mathrm{LJ}_{98}$ global minimum is particularly difficult to find. The origins of this difficulty are probably similar to the other non-icosahedral clusters. Analyses of the PESs of $\mathrm{LJ}_{38}$ and $\mathrm{LJ}_{75}$ using disconnectivity graphs have shown that they consist of a wide icosahedral "funnel" 31,32 and a much narrower 
funnel leading to the global minimum [33,34]. On relaxation down the PES the cluster is much more likely to enter the icosahedral funnel, where it is then trapped because of the large (free) energy barriers to escape from this funnel into the funnel of the global minimum.

This situation is compounded by the thermodynamics of these clusters 35.36]. The icosahedral funnel has a larger entropy because of the larger number of lowenergy minima, and so the funnel of the global minimum is only lowest in free energy at low temperatures. Therefore, at temperatures where the dynamics occur at a reasonable rate there is a thermodynamic driving force to enter the icosahedral funnel. For $\mathrm{LJ}_{98}$ there are at least 114 minima that are lower in energy than the second lowest-energy minimum in the tetrahedral funnel, and so the global minimum is only lowest in free energy below $T=0.0035 \epsilon k^{-1}$ (a typical melting temperature for a LJ cluster is $\left.0.3 \epsilon k^{-1}\right)$. This transition temperature is markedly lower than for $\mathrm{LJ}_{38}$ [34] or $\mathrm{LJ}_{75}$ [24].

The basin-hopping transformation of the PES helps to ameliorate some of these difficulties. The transformation changes the thermodynamics so that the global minimum still has a significant occupation probability at temperatures where the cluster can escape from the icosahedral funnel. However, on relaxation down the PES the system is still much more likely to enter the icosahedral funnel. For example, our optimization runs were fifteen times more likely to terminate at the lowest-energy $\mathrm{LJ}_{98}$ icosahedral minimum than at the global minimum.

Coordinate files for the new $\mathrm{LJ}_{98}$ structure, as well as all other putative LJ microcluster global optima, can be found in the Cambridge Cluster Database [37].

[1] D. J. Wales and H. A. Scheraga, Science in press (1999).

[2] L. T. Wille, Lennard-Jones clusters and the multipleminima problem, to appear.

[3] J. Farges, M. F. de Feraudy, B. Raoult, and G. Torchet, J. Chem. Phys. 84, 3491 (1986).

[4] O. Echt, K. Sattler, and E. Recknagel, Phys. Rev. Lett. 47, 1121 (1981).

[5] I. A. Harris, R. S. Kidwell, and J. A. Northby, Phys. Rev. Lett. 53, 2390 (1984).

[6] J. A. Northby, J. Chem. Phys. 87, 6166 (1987).

[7] A. L. Mackay, Acta Cryst. 15, 916 (1962).

[8] C. J. Tsai and K. D. Jordan, J. Phys. Chem. 97, 11227 (1993).
[9] F. H. Stillinger, Phys. Rev. E 59, 48 (1999).

[10] L. T. Wille, Chem. Phys. Lett. 133, 405 (1987).

[11] J. A. Niesse and H. R. Mayne, J. Chem. Phys. 105, 4700 (1996).

[12] D. M. Deaven, N. Tit, J. R. Morris, and K. M. Ho, Chem. Phys. Lett. 256, 195 (1996).

[13] M. D. Wolf and U. Landman, J. Phys. Chem. A 102, 6129 (1998).

[14] J. Kostrowicki, L. Piela, B. J. Cherayil, and H. A. Scheraga, J. Phys. Chem. 95, 4113 (1991).

[15] J. Pillardy and L. Piela, J. Phys. Chem. 99, 11805 (1995).

[16] G. L. Xue, J.Global Optimization 4, 425 (1994).

[17] M. R. Hoare and P. Pal, Adv. Phys. 20, 161 (1971).

[18] R. H. Leary, J. Global Optimization 11, 35 (1997).

[19] C. Barrón, S. Gómez, and D. Romero, Appl. Math. Lett. 9, 75 (1996).

[20] T. Coleman and D. Shalloway, J. Global Optimization 4, 171 (1994).

[21] C. Barrón, S. Gómez, and D. Romero, Appl. Math. Lett. 10, 25 (1997).

[22] J. P. K. Doye, D. J. Wales, and R. S. Berry, J. Chem. Phys. 103, 4234 (1995).

[23] J. P. K. Doye and D. J. Wales, Chem. Phys. Lett. 247, 339 (1995).

[24] D. J. Wales and J. P. K. Doye, J. Phys. Chem. A 101, 5111 (1997).

[25] C. L. Cleveland et al., Phys. Rev. Lett. 79, 1873 (1997).

[26] E. K. Parks, G. C. Niemann, K. P. Kerns, and S. J. Riley, J. Chem. Phys. 107, 1861 (1997).

[27] J. P. K. Doye and D. J. Wales, New J. Chem. in press (1998).

[28] A. P. Sutton and J. Chen, Phil. Mag. Lett. 61, 139 (1990).

[29] R. H. Leary, in preparation.

[30] D. J. Wales, personal communication.

[31] P. E. Leopold, M. Montal, and J. N. Onuchic, Proc. Natl. Acad. Sci. USA 89, 8271 (1992).

[32] J. D. Bryngelson, J. N. Onuchic, N. D. Socci, and P. G. Wolynes, Proteins: Structure, Function and Genetics 21, 167 (1995).

[33] J. P. K. Doye, M. A. Miller, and D. J. Wales, J. Chem. Phys. 110, 6896 (1999).

[34] J. P. K. Doye, M. A. Miller, and D. J. Wales, J. Chem. Phys. submitted (cond-mat/9903305).

[35] J. P. K. Doye and D. J. Wales, Phys. Rev. Lett. 80, 1357 (1998).

[36] J. P. K. Doye, D. J. Wales, and M. A. Miller, J. Chem. Phys. 109, 8143 (1998).

[37] D. J. Wales, J. P. K. Doye, A. Dullweber and F. Y. Naumkin, The Cambridge Cluster Database, URL http://brian.ch.cam.ac.uk/CCD.htm. 